\title{
A NOTE ON RIESZ OPERATORS
}

\author{
C. K. CHUI, P. W. SMITH AND J. D. WARD
}

\begin{abstract}
The purpose of this note is to settle a problem posed by Caradus, Pfaffenberger, and Yood; namely, it is proved that every Riesz operator $R$ on a Hilbert space has a decomposition $R=C+Q$ where $C$ is compact and both $Q$ and $C Q-Q C$ are quasinilpotent.
\end{abstract}

Let $H$ denote a complex, separable, infinite dimensional Hilbert space. In [5], West showed that every Riesz operator was a decomposable Riesz operator, i.e., $R=C+Q$ where $C$ is a compact operator and $Q$ is quasinilpotent. In general, this decomposition is not unique.

A Riesz operator is said to be fully decomposable if $R$ is decomposable and, in addition, $C$ commutes with $Q$ for some decomposition $C$ and $Q$.

In [1, p. 58], an example of Gillespie and West was given showing that there are some Riesz operators on $H$ which are not fully decomposable. They produced a Riesz operator $R$ for which no decomposition could commute. This leads to the following question proposed in [1, p. 59]: Can every Riesz operator be decomposed in such a manner that the commutator $C Q-Q C$ is quasinilpotent? The purpose of this note is to give a positive answer to this question, and, in fact, a slightly stronger result is proved.

The key to our proof is a lemma of Gohberg and Krein which was stated without proof in [3, p. 17] and was later stated and proved by Stampfli [4].

Lemma 1 [Gohberg-Krein, Stampfli]. Let $E$ be a closed set in C. Let $\sigma(T) \backslash E$ consist of isolated points $\left\{\lambda_{j}\right\}$ which of necessity cluster only on E. Let each $\lambda_{j}$ be a point of finite multiplicity. Then, $T=S+K$ where $K$ is compact and $\sigma(S) \subset E$.

Our theorem will depend heavily on the "Stampfli decomposition" and on its notation. Let us recall the pertinent steps. It was shown by Stampfli [4] that for a $T$ satisfying the hypotheses of Lemma 1,

Received by the editors March 5, 1976.

AMS (MOS) subject classifications (1970). Primary 47B05.

Key words and phrases. Riesz operators, quasinilpotent operators. 


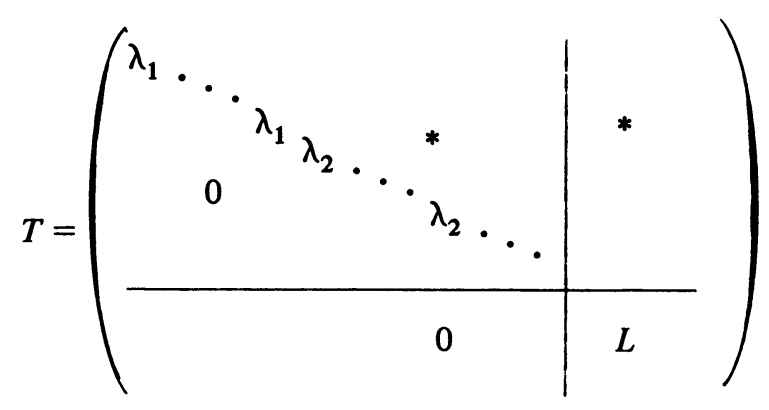

That is, if

$$
P_{j}=\frac{1}{2 \pi i} \int_{\left|\lambda-\lambda_{j}\right|=\varepsilon_{j}}(\lambda-T)^{-1} d \lambda
$$

where $0<\varepsilon_{j}<\min \left\{\min _{i \neq j}\left(\left|\lambda_{i}-\lambda_{j}\right|\right)\right.$, $\left.\operatorname{dist}\left(\lambda_{j}, E\right)\right\}$ for $i \neq j$, then $T$ has the matrix form listed above, where $L=Q T Q$ and $Q$ is the orthogonal projection on $\left(\sum P_{j} H\right)^{\perp}$. Now let $\left\{\alpha_{k}\right\}$ be a countable dense subset of $E$. With each $\lambda_{j}$, associate an $\alpha_{k_{j}}$ as follows. Choose $\alpha_{k_{j}}$ such that $\left|\alpha_{k_{j}}-\lambda_{j}\right| \leqslant 2 \operatorname{dist}\left(\lambda_{j}, E\right)$. For simplicity write $\alpha_{k_{j}}$ as $\alpha_{j}$. Next set

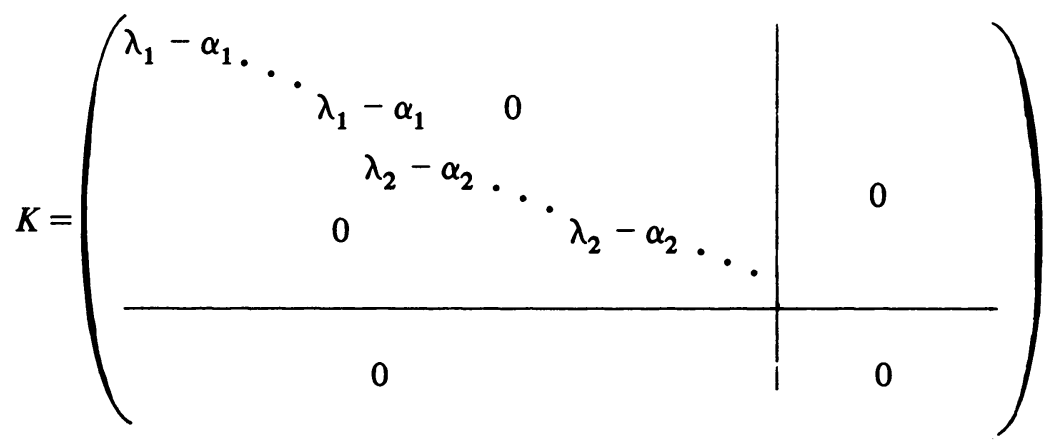

and define $S=T-K$. Obviously, $K$ is compact.

We are now ready to state our theorem.

THEOREM 1. Let $T$ satisfy the hypotheses of Lemma 1. Then in the "Stampfli decomposition" $T=S+K$, the commutator $S K-K S$ is a compact quasinilpotent operator.

Proof. Clearly $S K-K S$ is compact. Using the above notation, it is easily seen that

$$
S K-K S=\left(\begin{array}{cc}
\hat{S} \hat{K}-\hat{K} \hat{S} & \hat{K}^{*} \\
0 & 0
\end{array}\right)
$$

where $\hat{K}^{*}$ denotes the product of $\hat{K}$ and the northeast block of $S$ and $\hat{S}$ denotes the northwest corner of $S$.

We first show that $\hat{S} \hat{K}-\hat{K} \hat{S}$, viewed as an operator on $\sum \overline{P_{j} H}$ is quasinilpotent. As a matrix $\hat{S} \hat{K}-\hat{K} \hat{S}$ is a compact operator, upper triangular with 
main diagonal identically zero. Let $K_{n}=P_{n}(\hat{S} \hat{K}-\hat{K} \hat{S}) P_{n}$ where $P_{n}$ projects onto $\operatorname{sp}\left\{e_{1}, \ldots, e_{n}\right\}$ where $\left\{e_{i}\right\}_{i=1}^{\infty}$ is the orthonormal basis for which $\hat{S}$ is upper triangular. Since $P_{n}$ converges to the identity in the strong operator topology, $K_{n}$ converges uniformly to $\hat{S} \hat{K}-\hat{K} \hat{S}$. Clearly, each $K_{n}$ is quasinilpotent (actually nilpotent), so by [3, Theorem 4.1], $\hat{S} \hat{K}-\hat{K} \hat{S}$, as a uniform limit of compact quasinilpotent operators, is quasinilpotent.

To complete the proof, it suffices to show that $S K-K S$ is quasinilpotent. By the Riesz spectral theorem for compact operators, this is equivalent to showing that $S K-K S$ has no nonzero eigenvalues.

So assume $\lambda \neq 0$ and

$$
\left(\begin{array}{cc}
\hat{S} \hat{K}-\hat{K} \hat{S} & \hat{K}^{*} \\
0 & 0
\end{array}\right)\left(\begin{array}{l}
e_{1} \\
e_{2}
\end{array}\right)=\lambda\left(\begin{array}{l}
e_{1} \\
e_{2}
\end{array}\right) .
$$

Upon equating components of the vectors, we see that

$$
e_{2}=0 \text { and }(\hat{S} \hat{K}-\hat{K} \hat{S}) e_{1}=\lambda e_{1}
$$

which is impossible; thus $S K-K S$ is quasinilpotent. This completes the proof.

Corollary. For a Riesz operator $R$ on a Hilbert space, we have $R=C+$ $Q$ where $C$ is compact and both $Q$ and $C Q-Q C$ are quasinilpotent.

\section{REFERENCES}

1. S. R. Caradus, W. E. Pfaffenberger and B. Yood, Calkin algebras and algebras of operators on Banach spaces, Lecture Notes in Pure and Appl. Math., no. 9, Dekker, New York, 1974.

2. T. A. Gillespie and T. T. West, A characterisation and two examples of Riesz operators, Glasgow Math. J. 9 (1968), 106-110. MR 38 \#5031.

3. I. C. Gohberg and M. G. Krein, Introduction to the theory of linear nonselfadjoint operators, "Nauka", Moscow, 1965; English transl., Transl. Math. Monographs, vol. 18, Amer. Math. Soc., Providence, R. I., 1969. MR 36 \#3137; 39 \#447.

4. J. G. Stampfli, Compact perturbations, normal eigenvalues and a problem of Salinas, J. London Math. Soc. (2) 9 (1974/75), 165-175. MR 51 \#1449.

5. T. T. West, The decomposition of Riesz operators, Proc. London Math. Soc. (3) 16 (1966), 737-752. MR 33 \#6417.

Department of Mathematics, Texas A\&M University, College Station, Texas 77843 